\title{
ON CERTAIN CLASSES OF P-VALENT FUNCTIONS
}

\author{
M. K. AOUF \\ Department of Mathematics \\ Faculty of Science \\ University of Mansoura \\ Mansoura, Egypt \\ (Received February 20, 1985)
}

ABSTRACT. Let $v_{k}^{\lambda}(\alpha, b, p)(k \geq 2, b \neq 0$ is any complex number, $0 \leq \alpha<p$ and $|\lambda|<\pi / 2)$ denote the class of functions $f(z)=z^{p}+\sum_{n=p+1}^{\infty} a_{n} z^{n}$ analytic in $U=\{z:|z|<1\}$ having $(p-1)$ critical points in $U$ and satisfying

$$
\underset{r \rightarrow 1^{-}}{\lim \sup _{0}} \int_{0}^{2 \pi}\left|\frac{\operatorname{Re}\left\{e^{i \lambda}\left[p+\frac{1}{b}\left(1+\frac{z f^{\prime \prime}(z)}{f^{\prime}(z)}-p\right)\right]-\alpha \cos \lambda\right\}}{p-\alpha}\right| d \theta \leq k \pi \cos \lambda \text {. }
$$

In this paper we generalize both those functions $f(z)$ which are $p$-valent convex of order $\alpha, \quad 0 \leq \alpha<p$, with bounded boundary rotation and those p-valent functions $f(z)$ for which $\mathrm{zf}^{\prime}(\mathrm{z}) / \mathrm{p}$ is $\lambda$-spirallike of order $\alpha, 0 \leq \alpha<\mathrm{p}$.

KEY WORDS AND PHRASES. p-Valent, starlike, convex, spirallike functions, functions with bounded boundary rotation.

AMS SUBJECT CLASSIFICATION CODE. 30A32, $30 \mathrm{~A} 36$.

1. INTRODUCTION.

Let $M_{k}(k \geq 2)$ denote the lower class of real-valued functions $m(t)$ of bounded variation on $[0,2 \pi]$ which satisfy the conditions $\int_{0}^{2 \pi} d m(T)=2$ and $\int_{0}^{2 \pi}|d m(t)| \leq k$.

Let $A_{p}$, where $p$ is a positive integer, denote the class of functions $f(z)=$ $z^{p}+\sum_{n=p+1}^{\infty} a_{n} z^{n}$ which are analytic in $U=\{z:|z|<1\}$. For $f \varepsilon A_{p}$, we say that $f$ belongs to the class $v_{k}^{\lambda}(\alpha, b, p)(k \geq 2, b \neq 0$ is any complex number, $0 \leq \alpha<p$ and $|\lambda|<\pi / 2)$ if there exists $\delta>0$ such that

$$
\int_{0}^{2 \pi} \operatorname{Re}\left\{1+\frac{\mathrm{re}^{i \theta} \mathrm{f}^{\prime \prime}(\mathrm{re})}{\mathrm{f}^{\prime}\left(\mathrm{re} \mathrm{e}^{i \theta}\right)}\right\} \mathrm{d} \theta=2 \mathrm{p} \pi \quad(1-\delta<\mathrm{r}<1)
$$

and

$$
\limsup _{r \rightarrow 1^{-}} \int_{0}^{2 \pi}\left|\frac{\operatorname{Re}\left\{e^{i \lambda}\left[p+\frac{1}{b}\left(1+\frac{z f^{\prime \prime}(z)}{f^{\prime}(z)}-p\right)\right]-\alpha \cos \lambda\right\}}{p-\alpha}\right| d \theta \leq k \pi \cos \lambda .
$$


condition (1.1) implies that $f$ has $(p-1)$ critical points in $U$.

It is noticed that, by giving specific values to $k, \alpha, b, p$ and $\lambda$ in $v_{k}^{\lambda}(\alpha, b, p)$, we obtain the following important subclasses studied by various authors in earlier papers:

(1) $v_{k}^{\lambda}(o, 1, p)=v_{k}^{\lambda}(p)$. is the class of $p$-valent functions investigated by Silvia [1].

(2) $\mathrm{v}_{\mathrm{k}}^{\mathrm{O}}(0,1,1)=\mathrm{V}_{\mathrm{k}}$, is the class of bounded boundary rotation introduced by Löwner [2] and Paatero [3, 4] $v_{k}^{0}(\alpha, 1,1)=,v_{k}(\alpha), 0 \leq \alpha<1$, is the class of functions $f(z) \in A_{1}$ studied by Padmanabhan and Parvatham [5], $v_{k}^{\lambda}(0,1,1)=v_{k}^{\lambda}$, is the class of functions $f(z) \varepsilon A_{1}$ investigated by Moulis [6] and Silvia [7], $v_{k}^{\lambda}(\alpha, 1,1)=$ $v_{k}^{\lambda}(\alpha), 0 \leq \alpha<1$, is the class of functions $f(z) \varepsilon A_{1}$ investigated by Moulis [8], $v_{k}^{o}(o, b, 1)=v_{k}(b)$, is the class of functions $f(z) \varepsilon A_{1}$ introduced by Nasr [9] and $\mathrm{v}_{\mathrm{k}}^{\lambda}(\alpha, b, 1)=\mathrm{v}_{\mathrm{k}}^{\lambda}(\alpha, \mathrm{b})$, is the class of functions $\mathrm{f}(\mathrm{z}) \in \mathrm{A}_{1}$ investigated by Lakshma[b,ll].

(3) $v_{2}^{o}(o, 1, p)=C(p)$, is the class of $p$-valent convex functions considered by Goodman [12], $v_{2}^{o}(\alpha, 1, p)=C(\alpha, p)$, is the class of $p$-valent convex functions of order $\alpha, o \leq \alpha<p, \quad v_{2}^{\lambda}(o, 1, p)=C^{\lambda}(p)$, is the class of functions $f(z) \in A_{p}$ for which $z f^{\prime}(z) / p$ is $\lambda$-spirallike in $U, v_{2}^{\lambda}(\alpha, 1, p)=c^{\lambda}(\alpha, p)$, is the class of functions $f(z) \varepsilon A_{p}$ for which $z f^{\prime}(z) / p$ is $\lambda$-spirallike of order $\alpha, 0 \leq \alpha<p, v_{2}^{o}(0, b, p)=$ $c(b, p)$, is the class of p-valent functions satisfying

$$
\operatorname{Re}\left[p+\frac{1}{b}\left(1+\frac{z f^{\prime \prime}(z)}{f^{\prime}(z)}-p\right)\right]>0, z \in U \text {. }
$$

The class $c(b, p)$ introduced by Aouf [13] and finally for k=2 any function $f(z) \varepsilon$ $\mathrm{v}_{2}^{\lambda}(\alpha, \mathrm{b}, \mathrm{p})=\mathrm{c}^{\lambda}(\alpha, \mathrm{b}, \mathrm{p})$ if and only if

$$
\operatorname{Re} e^{i \lambda}\left[p+\frac{1}{b}\left(1+\frac{z f^{\prime \prime}(z)}{f^{\prime}(z)}-p\right)\right]>\alpha \cos \lambda, z \in U \text {. }
$$

Also from $v_{k}^{\lambda}(\alpha, b, p)$, we can obtain the following important subclasses:

(4) $v_{k}^{o}(\alpha, 1, p)=v_{k}(\alpha, p)$, is the class of p-valent functions $f(z) \varepsilon A_{p}$ satisfying

$$
\lim _{\mathrm{r} \rightarrow 1^{-}} \sup \int_{0}^{2 \pi}\left|\frac{\operatorname{Re}\left\{1+\frac{z f^{\prime \prime}(z)}{f^{\prime}(z)}\right\}-\alpha}{p-\alpha}\right| d \theta \leq k \pi \text {, }
$$

i.e., $f(z) \varepsilon V_{k}(\alpha, p)$ if and only if

$$
f^{\prime}(z)=p z^{p-1}\left\{\exp -(p-\alpha) \int_{0}^{2 \pi} \log \left(1-z e^{-i t}\right) \operatorname{dm}(t)\right\}, m(t) \varepsilon M_{k} .
$$


(5) $\mathrm{v}_{\mathrm{k}}^{\lambda}(\alpha, 1, \mathrm{p})=\mathrm{v}_{\mathrm{k}}^{\mathrm{O}}\left(\alpha, \cos \mathrm{e}^{-i \lambda}, \mathrm{p}\right)=\mathrm{v}_{\mathrm{k}}^{\lambda}(\alpha, \mathrm{p}),|\lambda|<\frac{\pi}{2}$, is the class of p-valent functions $f(z) \in A_{p}$ satisfying

$$
\lim _{\mathrm{r}^{-} 1^{-}} \sup \int_{0}^{2 \pi}\left|\frac{\operatorname{Re}\left\{\mathrm{e}^{i \lambda}\left(1+\frac{z \mathrm{f}^{\prime \prime}(z)}{\mathrm{f}^{\prime}(z)}\right)\right\}-\alpha \cos \lambda}{p-\alpha}\right| \mathrm{d} \theta \leq \mathrm{k} \pi \cos \lambda
$$

i.e., $f(z) \varepsilon V_{k}^{\lambda}(\alpha, p)$ if and only if

$f^{\prime}(z)=p z^{p-1} \exp \left\{-(p-\alpha) \cos \lambda e^{-i \lambda} \int_{0}^{2 \pi} \log \left(1-z e^{-i t}\right) d m(t)\right\}, m(t) \varepsilon M_{k} \cdot$

(6) $v_{k}^{o}(o, b, p)=v_{k}(b, p)$, is the class of p-valent functions $f(z) \varepsilon A_{p}$ satisfying

$$
\lim _{r \rightarrow 1^{-}} \sup \int_{0}^{2 \pi}\left|\operatorname{Re}\left\{p+\frac{1}{b}\left(1+\frac{z f^{\prime \prime}(z)}{f^{\prime}(z)}-p\right)\right\}\right| d \theta \leq k \pi p,
$$

i.e., $f(z) \varepsilon V_{k}(b, p)$ if and only if

$$
f^{\prime}(z)=p z^{p-1} \exp \left\{-p b \int_{0}^{2 \pi} \log \left(1-z e^{-i t}\right) \operatorname{dm}(t)\right\}, m(t) \varepsilon M_{k} \cdot
$$

(7) $v_{k}^{o}(\alpha, b, p)=v_{k}(\alpha, b, p)$, is the class of p-valent functions $f(z) \in A_{p}$ satisfying

$$
\lim _{\mathrm{r}^{-} 1^{-}} \sup _{0} \int_{0}^{2 \pi}\left|\frac{\operatorname{Re}\left\{\mathrm{p}+\frac{1}{b}\left(1+\frac{z f^{\prime \prime}(z)}{f^{\prime}(z)}-p\right)\right\}-\alpha}{p-\alpha}\right| d \theta \leq k \pi,
$$

i.e., $f(z) \varepsilon V_{k}(\alpha, b, p)$ if and only if

$$
f^{\prime}(z)=p z^{p-1} \exp \left\{-(p-\alpha) b \int_{0}^{2 \pi} \log \left(1-z e^{-i t}\right) \operatorname{dm}(t)\right\}, m(t) \varepsilon M_{k}
$$

We state below some lemmas that are needed in our investigation.

LEMMA 1 [10]. $h(z) \varepsilon v_{k}^{\lambda}(\alpha, b)$ if and only if

(1) $h^{\prime}(z)=\exp \left\{-(1-\alpha) b \cos \lambda e^{-i \lambda} \int_{0}^{2 \pi} \log \left(1-z e^{-i t}\right) d m(t)\right\}, m(t) \varepsilon M_{k}$.

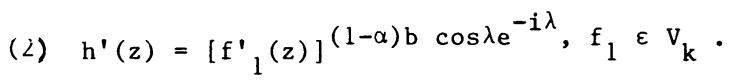

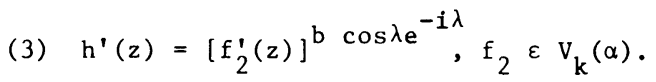

(4) $h^{\prime}(z)=\left[f_{3}^{\prime}(z)\right]^{(1-\alpha) b}, f_{3} \varepsilon v_{k}^{\lambda}$.

(5) $h^{\prime}(z)=\left[f_{4}^{\prime}(z)\right]^{b}, f_{4} \varepsilon v_{k}^{\lambda}(\alpha)$.

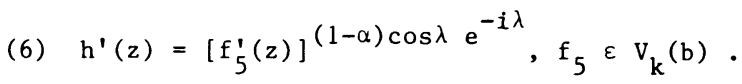

(7) there exists two normalized starlike functions $s_{1}(z)$ and $s_{2}(z)$ such that

$$
f^{\prime}(z)=\left\{\frac{\left[\frac{s_{1}(z)}{z}\right]}{\frac{k+2}{4}}(1-\alpha) b \cos \lambda e^{-i \lambda}\right.
$$


LEMMA $2[10]$. If $h(z)$ belongs to $v_{k}^{\lambda}(\alpha, b)$, then $H(z)$ is defined by

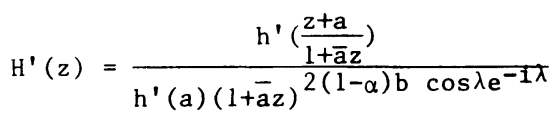

$H(o)=0,|z|<1,|a|<1$ is also in the class $v_{k}^{\lambda}(\alpha, b)$.

LEMMA $3[10]$. Suppose $h(z)=z+b_{2} z^{2}+b_{3} z^{z}+\ldots$ belongs to $v_{k}^{\lambda}(\alpha, b)$ then

$$
\left|b_{2}\right| \leq \frac{k}{2}(1-a)|b| \cos \lambda \text {, }
$$

and

$$
\left|b_{3}\right| \leq \frac{1}{3}(1-\alpha)|b| \cos \lambda\left[1-(1-\alpha)|b| \cos \lambda \frac{k^{2}}{2}\right] .
$$

These bounds are sharp, with equality for the function $h(z) \varepsilon v_{k}^{\lambda}(\alpha, b)$ defined by

$$
h^{\prime}(z)=\left[\frac{(1-z)^{\frac{k}{2}-1}}{(1+z)^{\frac{k}{2}+1}}\right]^{(1-\alpha) b \cos \lambda e^{-i \lambda}} .
$$

LEMMA $4[1]$. $g(z) \varepsilon V_{k}^{\lambda}(p), p \geq 1$, if and only if $g^{\prime}(z)=p z^{p-1}\left[f_{3}^{\prime}(z)\right]^{p}$ for some $\mathrm{f}_{3} \varepsilon \mathrm{V}_{\mathrm{k}}^{\lambda}$.

2. REPRESENTATION FORMULAS FOR THE CLASS $\mathrm{v}_{\mathrm{k}}^{\lambda}(\alpha, \mathrm{b}, \mathrm{p})$.

LEMMA 5. $\mathrm{f}(\mathrm{z}) \quad \mathrm{V}_{\mathrm{k}}^{\lambda}(\alpha, \mathrm{b}, \mathrm{p})$ if and only if

$$
f^{\prime}(z)=p z^{p-1}\left[h^{\prime}(z)\right]^{\frac{(p-\alpha)}{1-\alpha}}=p z^{p-1}+{ }_{n=p+1}^{\infty} n a_{n} z^{n-1}
$$

for some $h \in v_{k}^{\lambda}(a, b)$.

PROOF. Let $f^{\prime}(z)=p z^{p-1}\left[h^{\prime}(z)\right]^{\left(\frac{p-\alpha}{1-\alpha}\right)}=p z^{p-1}+n_{n=p+1}^{\infty} n a_{n} z^{n-1}$ for $h(z)=z+\sum_{n=2}^{\infty} b_{n} z^{n} \varepsilon v_{k}^{\lambda}(\alpha, b), z \varepsilon U$.

By direct computation, we obtain

$$
\begin{aligned}
& \int_{0}^{2 \pi}\left|\frac{\operatorname{Re}\left\{e^{i \lambda}\left[p+\frac{1}{b}\left(1+\frac{z f^{\prime \prime}(z)}{f^{\prime}(z)}-p\right)\right]-\alpha \cos \lambda\right\}}{p-\alpha}\right| d \theta= \\
& \int_{0}^{2 \pi}\left|\frac{\operatorname{Re}\left\{e^{i \lambda}\left[1+\frac{1}{b} \frac{z h^{\prime \prime}(z)}{h^{\prime}(z)}\right]-\alpha \cos \lambda\right\}}{1-\alpha}\right| d \theta
\end{aligned}
$$

and the result follows from (1.2).

An immediate consequence of Lemmas 5 and 1 is

THEOREM 1. $\mathrm{f}(\mathrm{z}) \varepsilon \mathrm{v}_{\mathrm{k}}^{\lambda}(\alpha, \mathrm{b}, \mathrm{p})$ if and only if

(1) $f^{\prime}(z)=p z^{p-1} \exp \left\{-(p-\alpha) b \cos \lambda e^{-i \lambda} \int_{0}^{2 \pi} \log \left(1-z e^{-i t} \cdot\right) d m(t)\right\}, m(t) \varepsilon M_{k}$. 


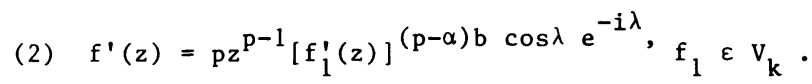

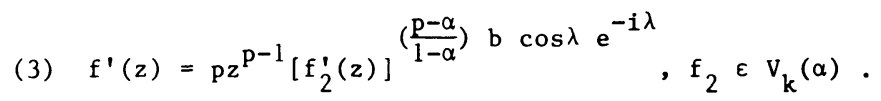

(4) $\quad f^{\prime}(z)=p z^{p-1}\left[f_{3}^{\prime}(z)\right]^{(p-\alpha) b}, f_{3} \varepsilon v_{k}^{\lambda}$.

(5) $f^{\prime}(z)=p z^{p-1}\left[f_{4}^{\prime}(z)\right]^{\left(\frac{p-\alpha}{1-\alpha}\right) b}, f_{4} \in v_{k}(\alpha)$

(6) $f^{\prime}(z)=p z^{p-1}\left[f_{5}^{\prime}(z)\right]^{(p-\alpha) \cos \lambda} e^{-i \lambda}, f_{5} \varepsilon v_{k}(b)$.

(7) there exists two normalized starlike functions $s_{1}(z)$ and $s_{2}(z)$ such that

$$
\begin{aligned}
f^{\prime}(z)=p z^{p-1} & \frac{\left\{\frac{s_{1}(z)}{z}\right\}^{\frac{k+2}{4}}(p-\alpha) b \cos \lambda e^{-1 \lambda}}{\left\{\frac{s_{2}(z)}{z}\right\}^{\frac{k-2}{4}}}
\end{aligned}
$$

Also an immediate consequence of Theorem 1 (4) and Lemma 4 is

THEOREM 2. $\mathrm{f}(\mathrm{z}) \in \mathrm{v}_{\mathrm{k}}^{\lambda}(\alpha, b, \mathrm{p})$ if and only if

$f^{\prime}(z)=p z^{p-1}\left[\frac{g^{\prime}(z)}{p z^{p-1}}\right]^{\left(\frac{p-\alpha}{p}\right) b}, g \in v_{k}^{\lambda}(p)$

3. COEFFICIENT ESTIMATES FOR THE CLASS $v_{k}^{\lambda}(\alpha, b, p)$.

THEOREM 3. If $\mathrm{f}(\mathrm{z})=z^{\mathrm{p}}+\sum_{\mathrm{n}=\mathrm{p}+1}^{\infty} a_{n} z^{\mathrm{n}} \varepsilon \mathrm{v}_{\mathrm{k}}^{\lambda}(\alpha, \mathrm{b}, \mathrm{p})$, then

$$
\begin{aligned}
& \left|a_{p+1}\right| \leq\left[\frac{p(p-\alpha)}{p+1}\right] k|b| \cos \lambda, \\
& \left|a_{p+2}\right| \leq p\left(\frac{p-\alpha}{p+2}\right)|b| \cos \lambda\left\{\left[1-(1-\alpha)|b| \cos \lambda \frac{k^{2}}{2}\right]+(p-1)|b| \cos \lambda \frac{k^{2}}{2}\right\}
\end{aligned}
$$

These bounds are sharp with equality for

$$
f_{*}^{\prime}(z)=p z^{p-1}\left[\frac{(1-z)^{\frac{k}{2}-1}}{(1+z)^{\frac{k}{2}}+1}\right](p-\alpha) b \cos \lambda e^{-i \lambda}
$$

PROOF. By Lemma 5, there exists an $h(z)=z+\sum_{n=2}^{\infty} b_{n} z^{n} \varepsilon v_{k}^{\lambda}(\alpha, b)$ such that

$f^{\prime}(z)=p z^{p-1}+\sum_{n=p+1}^{\infty} n a_{n} z^{n-1}=p z^{p-1}\left[1+\sum_{n=2}^{\infty} n b_{n} z^{n-1}\right]\left(\frac{p-\alpha}{1-\alpha}\right)$

Expanding the right side of $(3.4)$, we obtain

$$
f^{\prime}(z)=p z^{p-1}+2 p\left(\frac{p-\alpha}{1-\alpha}\right) b_{2} z^{p}+p\left\{3\left(\frac{p-\alpha}{1-\alpha}\right) b_{3}+2\left(\frac{p-\alpha}{1-\alpha}\right)\left(\frac{p-1}{1-\alpha}\right) b_{2}{ }^{2}\right\} z^{p+1}+\ldots
$$

Equating coefficents from (3.5) and (3.4), we have 


$$
\begin{aligned}
& (p+1) a_{p+1}=2 p\left(\frac{p-\alpha}{1-\alpha}\right) b_{2}, \\
& (p+2) a_{p+2}=p\left\{3\left(\frac{p-\alpha}{1-\alpha}\right) b_{3}+2\left(\frac{p-\alpha}{1-\alpha}\right)\left(\frac{p-1}{1-\alpha}\right) b_{2}{ }^{2}\right\} .
\end{aligned}
$$

Thus the result follows from lemma 3 .

4. SHARP RADIUS OF CONVEXITY OF THE CLASS $v_{k}^{\lambda}(\alpha, b, p)$.

LEMMA 6. If $\mathrm{f} \varepsilon \mathrm{V}_{\mathrm{k}}^{\lambda}(\alpha, \mathrm{b}, \mathrm{p})$, then the transformation $\mathrm{F}_{\mathrm{a}}$ satisfying

$$
F_{a}^{\prime}(z)=\frac{p a^{p-1} z^{p-1} f^{\prime}\left(\frac{z+a}{1+\bar{a} z}\right)}{f^{\prime}(a)(z+a)^{p-1}(1+\bar{a} z)^{2(p-\alpha) b \cos \lambda e^{-i \lambda}-p+1}}, \quad z \in U
$$

and $\mathrm{F}_{\mathrm{a}}(\mathrm{o})=\mathrm{o}$, is in $\mathrm{V}_{\mathrm{k}}^{\lambda}(\alpha, \mathrm{b}, \mathrm{p})$ for all $|\mathrm{a}|<1$.

The proof of this lemma follows by using lemmas 5 and 2 .

LEMMA 7. For $|z| \leq r$ and $f$ ranging over $v_{k}^{\lambda}(\alpha, b, p)$, the domain of values of $\frac{z f^{\prime \prime}(z)}{f^{\prime}(z)}$ is the disc with center

$$
\left(\frac{\left[2(p-\alpha) \operatorname{Re}\left\{b \cos \lambda e^{-i \lambda}\right\}-p+1\right] r^{2}+(p-1)}{1-r^{2}}, \frac{2(p-\alpha) \operatorname{Im}\left\{b \cos \lambda e^{-1 \lambda}\right\} r^{2}}{1-r^{2}}\right)
$$

and radius $\frac{p(p-\alpha) k|b| \cos \lambda r}{1-r^{2}}$.

PROOF. Whenever

$$
f(z)=z^{p}+\sum_{n=p+1}^{\infty} a_{n} z^{n} \in v_{k}^{\lambda}(\alpha, b, p), \quad \lim _{z \rightarrow 0}\left\{\frac{f^{\prime \prime}(z)-p(p-1) z^{p-2}}{z^{p-1}}\right\}=p(p-1) a_{p+1}
$$

For $f \varepsilon v_{k}^{\lambda}(\alpha, b, p)$, let $F_{a}(z)=z^{p}+\sum_{n=p+1}^{\infty} A_{n} z^{n} \varepsilon v_{k}^{\lambda}(\alpha, b, p)$ be given by Lemma 6 for $|a|<1$. By direct calculation we have

$$
p(p+1) A_{p+1}=p\left(1-|a|^{2}\right) \frac{f^{\prime \prime}(a)}{f^{\prime}(a)}-\frac{p\left[2(p-\alpha) b \cos \lambda e^{-1 \lambda}-p+1\right]|a|^{2}+p(p-1)}{a} .
$$

Combining (3.1) and (4.2), we obtain

$$
\left|\frac{f^{\prime \prime}(a)}{f^{\prime}(a)}-\frac{\left[2(p-\alpha) b \cos \lambda e^{-i \lambda}-p+1\right]|a|^{2}+(p-1)}{a\left(1-|a|^{2}\right)}\right| \leq \frac{p(p-\alpha) k|b| \cos \lambda}{\left(1-|a|^{2}\right)}
$$

From (4.3) it follows that for $|z|=r<1$,

$$
\left|\frac{z f^{\prime \prime}(z)}{f^{\prime}(z)}-\frac{\left[2(p-\alpha) b \cos \lambda e^{-i \lambda}-p+1\right] r^{2}+(p-1)}{\left(1-r^{2}\right)}\right| \leq \frac{p(p-\alpha) k|b| \cos \lambda r}{\left(1-r^{2}\right)}
$$

and the proof is completed.

THEOREM 4. The sharp radius of convexity of the class $v_{k}^{\lambda}(\alpha, b, p)$ is

$$
\begin{aligned}
& r_{0}=2\left\{(p-\alpha) k|b| \cos \lambda+\left[(p-\alpha)^{2} k^{2}|b|^{2} \cos ^{2} \lambda-4\left(2\left(1-\frac{\alpha}{p}\right) \operatorname{Re}\left\{b \cos \lambda e^{-i \lambda}\right\}-1\right)\right]^{\frac{1}{2}}\right\}^{-1} \\
& \text { PROOF. From (4.4), we have }
\end{aligned}
$$




$$
\left|\left(1+\frac{z f^{\prime \prime}(z)}{f^{\prime}(z)}\right)-p\left(\frac{1+\left(2\left(1-\frac{\alpha}{p}\right) b \cos \lambda e^{-i \lambda}-1\right) r^{2}}{1-r^{2}}\right)\right| \leq \frac{p(p-\alpha) k|b| \cos \lambda r}{1-r^{2}}
$$

which implies that

$$
\operatorname{Re}\left\{1+\frac{z f^{\prime \prime}(z)}{f^{\prime}(z)}\right\} \geq p\left[\frac{1-(p-\alpha) k|b| \cos \lambda r+\left(2\left(1-\frac{\alpha}{p}\right) \operatorname{Re}\left\{b \cos \lambda e^{-i \lambda}-1\right) r^{2}\right.}{1-r^{2}}\right] .
$$

Therefore $\operatorname{Re}\left\{1+\frac{z f^{\prime \prime}(z)}{f^{\prime}(z)}\right\}>0$ for $|z|<r_{0}$ is given by (4.5). The function $f_{x}(z)$ defined by

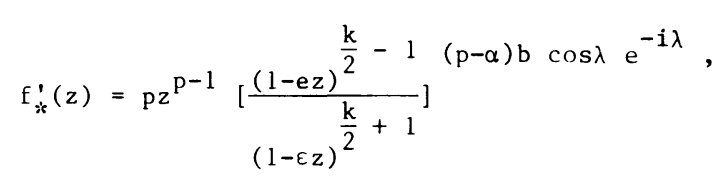

$$
\text { when } z=r, e=\frac{r+p e^{i \lambda} \sqrt{\frac{b}{b}}}{1+r p e^{i \lambda} \sqrt{\frac{b}{b}}} \text { and } \varepsilon=\frac{r-p e^{i \lambda \sqrt{\frac{b}{b}}}}{1-r p e^{i \lambda} \sqrt{\frac{b}{b}}}
$$

shows that the bound in (4.5) is sharp.

5. DistORTION AND ROTATION THEOREMS FOR THE CLASS $v_{k}^{\lambda}(\alpha, b, p)$.

In [11] Lakshma used variational method to solve the extremal problems for the class $\mathrm{v}_{\mathrm{k}}^{\lambda}(\alpha, b)$. He proved the following:

LEMMA $8[11]$. Let $\xi \neq \circ$ be a given point in $U$ and let $H\left(x_{1}, x_{2}, \ldots x_{n+1}\right)$ be

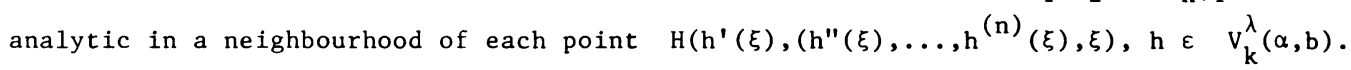
Then the functional

$$
J\left(h^{\prime}\right)=\operatorname{Re} H\left(h^{\prime}(\xi), h^{\prime \prime}(\xi), \ldots, h^{(n)}(\xi), \xi\right)
$$

attains its maximum (or minimum) in $v_{k}^{\lambda}(\alpha, b)$ only for a function of the form

$$
h^{\prime}(z)=\prod_{j=1}^{M}\left(1-\varepsilon_{j}\right)^{\beta} j^{(1-\alpha) b \cos \lambda e^{-i \lambda}} \quad{ }_{j=1}^{N}\left(1-e_{j} z\right)^{-\alpha_{j}(1-\alpha) b \cos \lambda e^{-i \lambda}}
$$

where $M \leq n, \quad N \leq n,\left|\varepsilon_{j}\right|=\left|e_{j}\right|=1, \sum_{j=1}^{M} \beta_{j} \leq \frac{k}{2}-1, \quad \sum_{j=1}^{N} \alpha_{j} \leq \frac{k}{2}+1$.

LEMMA $9[1]$. If $h(z) \varepsilon v_{k}^{\lambda}(\alpha, b)$, then we have

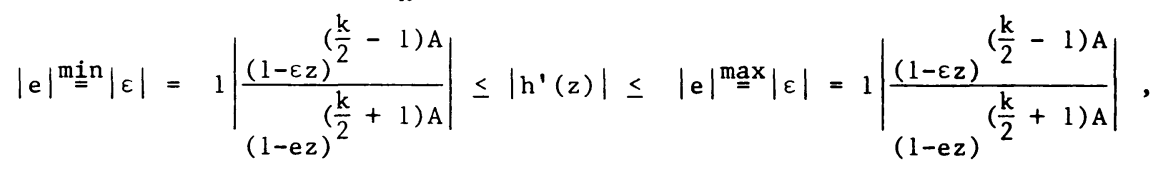

where $A=(1-\alpha) b \cos \lambda e^{-i \lambda}$.

Bounds are sharp with properly selected $e$ and $\varepsilon$ with $M=N=1$ in (5.1).

LEMMA $10[11]$. If $h(z) \varepsilon v_{k}^{\lambda}(\alpha, b)$, then for $|z|=r<1$, we obtain

$$
\left|\arg h^{\prime}(z)\right| \leq(1-\alpha) k|b| \cos \lambda \operatorname{arc} \sin |z| \text {. }
$$


The proof of the following two theorems follows from Lemma 5 and the above bounds. THEOREM 5. If $\mathrm{f} \varepsilon \mathrm{v}_{\mathrm{k}}^{\lambda}(\alpha, \mathrm{b}, \mathrm{p})$, then for $|\mathrm{z}|=\mathrm{r}<1$, we obtain

$$
|e|^{\min }|\varepsilon|=1\left|\frac{(1-\varepsilon z)^{\left(\frac{k}{2}-1\right) B}}{(1-e z)}\right| \leq\left|\frac{f^{\prime}(z)}{\left.p^{p-1}+1\right) B}\right| \leq|e|^{\max }|\varepsilon|=1\left|\frac{(1-\varepsilon z)^{\left(\frac{k}{2}-1\right) B}}{\left(\frac{k}{2}+1\right) B}\right| \text {, }
$$

where $B=(p-\alpha) b \cos \lambda e^{-i \lambda}$.

Bounds are sharp with equality for $f^{\prime}(z)=p z^{p-1}\left[h^{\prime}(z)\right]^{\left(\frac{p-\alpha}{1-\alpha}\right)}$, where $h(z)$ is defined by (5.1) with properly selected $e$ and $\varepsilon$ with $M=N=1$.

THEOREM 6. If $\mathrm{f} \varepsilon \mathrm{v}_{\mathrm{k}}^{\lambda}(\alpha, \mathrm{b}, \mathrm{p})$, then for $|\mathrm{z}|=\mathrm{r}<1$, we obtain

$$
\left|\arg f^{\prime}(z)\right| \leq(p-1)^{\theta}+(p-\alpha) k|b| \cos \lambda \operatorname{arc} \sin |z|
$$

6. HARDY CLASSES FOR THE CLASS $v_{k}^{\lambda}(\alpha, b)$.

In order to obtain the $H^{\mu}$ classes for the class $v_{k}^{\lambda}(\alpha, b)$, we will use the following lemmas.

LEMMA 11[7]. IF $\mathrm{f}_{3} \in \mathrm{V}_{\mathrm{k}}^{\lambda}$. Then for $|\mathrm{z}|=\mathrm{r},\left|\arg \mathrm{f}_{3}^{\prime}\left(\mathrm{re}^{i \theta}\right)\right| \leq \mathrm{k} \cos \lambda \operatorname{arc} \sin \mathrm{r}$. LEMMA $12[14]$. If $f^{\prime} \varepsilon H^{\mu}(0<\mu<1)$ then $f \in H^{\frac{\mu}{1-\mu}}$ where, for $\mu=1, H^{\infty}$ is the class of bounded functions.

LEMMA 13[15]. If $\mathrm{f}(\mathrm{z}) \varepsilon \mathrm{H}^{\mu}(0<\mu<1)$ and $\mathrm{f}(\mathrm{z})=\sum_{\mathrm{n}=0}^{\infty} a_{n^{2}} \mathrm{z}^{\mathrm{n}}$, then $a_{n}=o\left(n^{\frac{1}{\mu}}-1\right)$. LEMMA $14[1]$. If $\mathrm{f}_{3} \varepsilon \mathrm{V}_{\mathrm{k}}^{\lambda}$, then $\mathrm{f}_{3}^{\prime} \varepsilon \mathrm{H}^{\mu}$ for all $\mu<\frac{2}{\cos ^{2} \lambda(\mathrm{k}+2)}$ and $\mathrm{f}_{3} \varepsilon \mathrm{H}^{n}$ for $n<\frac{2}{\left[\cos ^{2} \lambda(k+2)-2\right]}, \cos ^{2} \lambda>\frac{2}{(k+2)} \cdot$ Furthermore, if $f_{3}^{\prime}$ is not of the form $\mathrm{f}_{3}^{\prime}(z)=\left[\mathrm{f}_{0}^{\prime}(z)\right]^{\cos \lambda \mathrm{e}^{-i \lambda}}$ where $\mathrm{f}_{0}$ is given by

$$
f_{0}^{\prime}(z)=\left(1-z e^{-i t} 0\right)^{-\left(\frac{k}{2}+1\right)} \exp \left\{\int_{0}^{2 \pi}-\log \left(1-z e^{-i t}\right) d m(t)\right\}
$$

(m(t) a probability measure on $[0,2 \pi])$, then there exists $\delta=\delta\left(f_{3}\right)>0$ and $\varepsilon=\varepsilon\left(f_{3}\right)>0$ such that

$\mathrm{f}_{3}^{\prime}(\mathrm{z}) \varepsilon \mathrm{H}^{\cos ^{2} \lambda(\mathrm{k}+2)}$ and $\mathrm{f}_{3} \varepsilon \mathrm{H}^{\left[\cos ^{2} \lambda(\mathrm{k}+2)-2\right]}$ for $\cos ^{2} \lambda>\frac{2}{(\mathrm{k}+2)}$.

THEOREM 7. If $h \varepsilon V_{k}^{\lambda}(\alpha, b)$, $\operatorname{Re}\{b\}>o$, then $h^{\prime} \varepsilon H^{\mu}$ for all

$$
\mu<\frac{2}{(1-\alpha) \operatorname{Re}\{b\} \cos ^{2} \lambda(k+2)} \text { and } h \varepsilon H^{n} \text { for } n<\frac{2}{\left[(1-\alpha) \operatorname{Re}\{b\} \cos ^{2} \lambda(k+2)-2\right]} \text {, }
$$


where $\cos ^{2} \lambda>\frac{2}{(1-\alpha) \operatorname{Re}\{b\}(k+2)}$. Furthermore, if $h^{\prime}$ is not of the form $h^{\prime}(z)=$

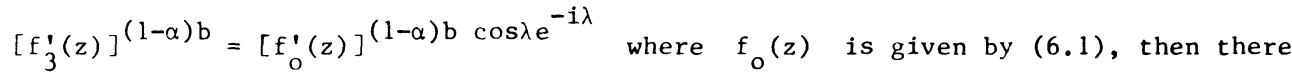
exists $\varepsilon=\varepsilon(h)>0$ and $\delta=\delta(h)>0$ such that

$$
h^{\prime} \varepsilon H^{\varepsilon+} \frac{2}{\left[(1-\alpha) \operatorname{Re}\{b\} \cos ^{2} \lambda(k+2)\right]} \text { and } h \varepsilon H^{\delta+} \frac{2}{\left[(1-\alpha) \operatorname{Re}\{b\} \cos ^{2} \lambda(k+2)-2\right]}
$$

for $\cos ^{2} \lambda>\frac{2}{(1-\alpha) \operatorname{Re}\{b\}(k+2)}$.

PROOF. If $h \in V_{k}^{\lambda}(\alpha, b)$, then it follows from Lemma $1(4)$ that

$$
h^{\prime}(z)=\left[f_{3}^{\prime}(z)\right]^{(1-\alpha) b}, f_{3} \varepsilon v_{k}^{\lambda}
$$

Then

$$
\left|h^{\prime}(z)\right|^{\mu}=\left|f_{3}^{\prime}(z)\right|^{\mu(1-\alpha) \operatorname{Re}\{b\}} \cdot \exp \left\{-\mu(1-\alpha) \operatorname{Im}\{b\} \arg f_{3}^{\prime}(z)\right\} .
$$

By Lemma 11, the exponential factor is bounded. Thus the result follows from Lemmas 12 and 14. From Theorem 7 and Lemma 13, we obtain a growth estimate for the Taylor's coefficients of $h \in v_{k}^{\lambda}(\alpha, b)$.

COROLlary 1. If $h(z)=z+\sum_{n=2}^{\infty} b_{n} z^{n} \varepsilon v_{k}^{\lambda}(\alpha, b)$ and $\cos ^{2} \lambda>\frac{2}{(1-\alpha) \operatorname{Re}\{b\}(k+2)}$, then

$$
b_{n}=o(n) \text {. }
$$

Acknowledgment. The author is thankful to Professor Dr. S. M. Shah for reading the manuscript and for helpful suggestions.

\section{REFERENCES}

1. SILVIA, E.M., A note of special classes of p-valent functions, Rocky Mt. J. Math. 9(2) (1979), 365-370.

2. LOWNER, K., Untersuchungen uber die Verzerrung bei konformen Abbildungen Einheitskreises $|z|<1$, die durch Funktionen mit nicht verschwindender Ableitung geliefert werden, Ber. Köngil. Sachs. Ces. Wiss. Leipzig 69 (1917),
89-106.

3. PAATERO, V., Uber die konforme Abbildung von Gelieten deren Rander von beschrankter Drehung sind, Ann. Acad. Sci. Fenn. Ser. A. (33) 9 (1931), 1-77.

4. PAATERO, V., Uner Gebiete von beschrankter Raddrehung, Ann. Acad. Sci. Fenn. Ser. A. 37 (1933), 1-20.

5. PADMANBHAN, K.S. and PARVATHAM, R., Properties of a class of functions with bounded boundary rotation, Ann. Polon. Math. 31 (3) (1975), 311-323.

6. MUULIS, E.J. Jr., A generalization of univalent functions with bounded bnundary rotation, Trans. Amer. Math. Soc., 174(1972), 369-381.

7. SILVIA, E.M., A variational method on certain classes of functions, Rev. Roum. Math.Pures et App1., 21(5) (1979), 549-557.

8. MOULIS, E.J. Jr., Generalizations of the Robertson functions, Pacific J. Math. 81(1) (1979), 167-174.

9. NASR, M.A., On a class of functions with bounded boundary rotation, Bul1. of the Institute of Math., Academia Sinica 5(1977), 27-36. 
10. LAKSHMA, R., On certain classes of functions with bounded boundary rotation, Indian J. Pure and Appl. Math., 13(2) (1982), 195-204.

11. LAKSHMA, R., A variational method on certain classes of analytic functions, Indian J. Pure and App1. Math., 14(5) (1983), 601-613.

12. GOODMAN, A.W., On the SchwarzpChristofell transformation and p-valent functions, Trans. Amer. Math. Soc., 68 (1950), 204-223.

13. AOUF, M.K., p-Valent classes related to convex functions of complex order, to appear, Rocky Mt.J. Math.

14. HARDY, G.H., and LITTLEWOOD, J.E., Some properties of fractional integrals II, Math. Z., 34(1932), 403-439.

15. DUREN, P.L., Theory of $\mathrm{H}^{\mathrm{P}}$ spaces, Pure and Appl. Math. 38 , Academic Press, New York, 1970. 


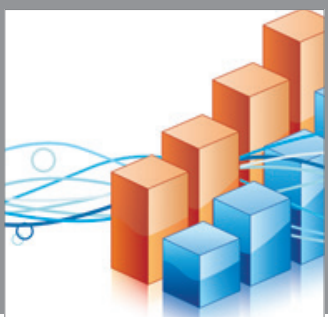

Advances in

Operations Research

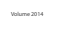

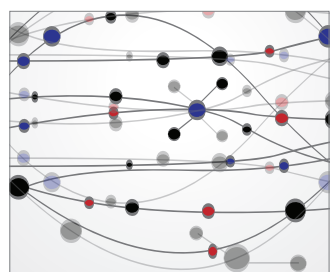

\section{The Scientific} World Journal
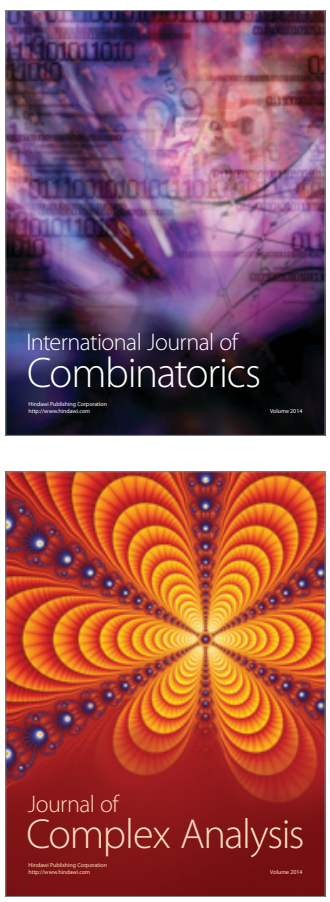

International Journal of

Mathematics and

Mathematical

Sciences
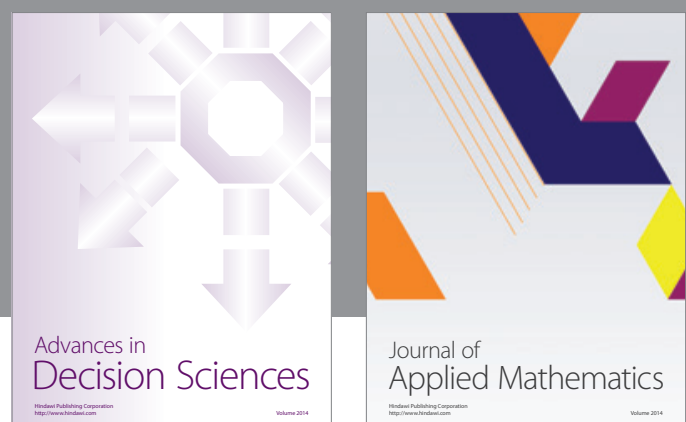

Journal of

Applied Mathematics
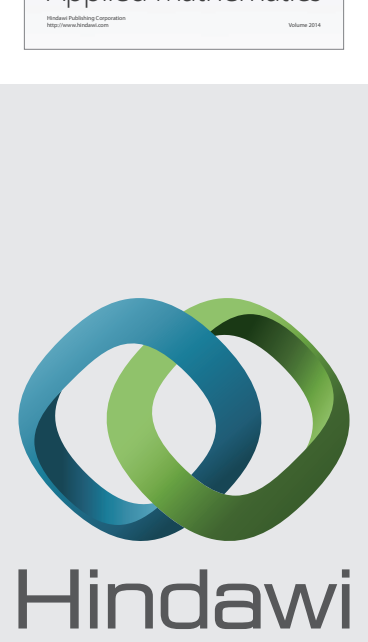

Submit your manuscripts at http://www.hindawi.com
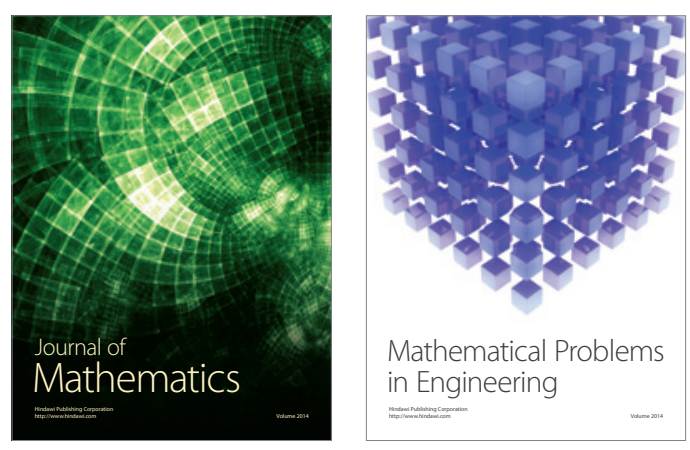

Mathematical Problems in Engineering
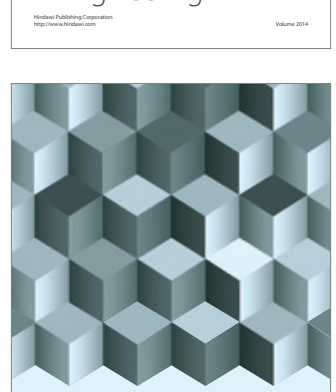

Journal of

Function Spaces
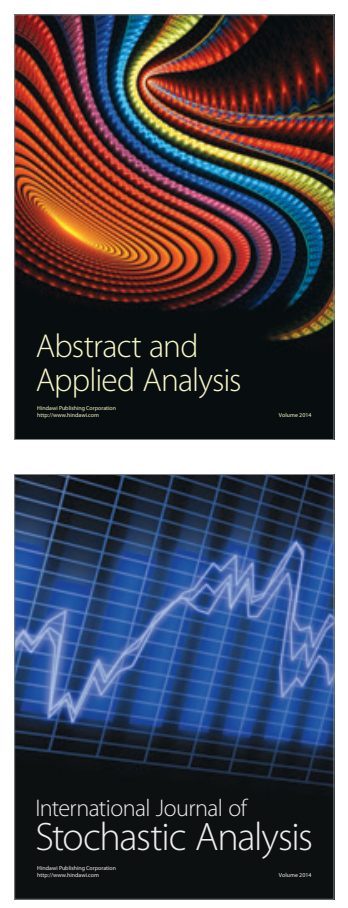

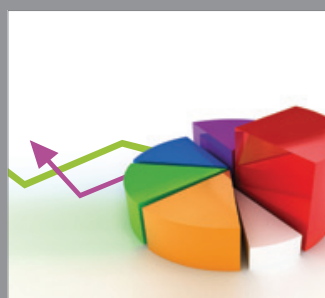

ournal of

Probability and Statistics

Promensencen
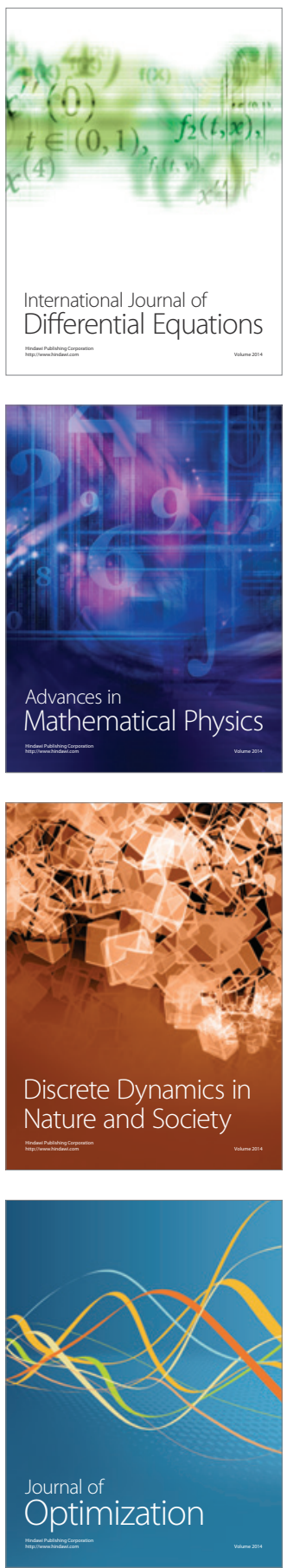